\title{
Numerical simulations of flow reversal in Rayleigh-Bénard convection
}

\author{
Roberto Benzi ${ }^{1}$ and Roberto Verzicco ${ }^{2}$ \\ 1 Dept. of Physics and INFN, University of Rome "Roma Tor Vergata", \\ Via della Ricerca Scientifica 1, 00133 Rome, Italy, ${ }^{2}$ DIMeG \& CEMeC, \\ Politecnico di Bari, Via Re David 200, 70125 Bari, Italy.
}

\begin{abstract}
We investigate numerically the statistical properties of the large scale flow in Rayleigh-Bénard convection. By using an external random perturbation on the temperature field, we were able to decrease the effective Prandtl number of the flow while keeping the Rayleigh number relatively small; this increases the Reynolds number thus making possible the numerical investigation of the longterm flow statistics. We also propose a simple and quantitative explanation for the experimental findings on the statistical distribution of flow reversals and reorientations.
\end{abstract}

Turbulent convection in a Rayleigh-Bénard cell is characterized by the complex behaviour of the unstable thermal boundary layers and plumes which produce a large scale (turbulent) flow in the cell. Experimentally, it has been observed that the large scale "wind" may exhibit abrupt flow reversals [1] and reorientations [2], [3], [4], whose statistical properties have been the subject of some theoretical investigations [1], [5], [8]. Up to now, there has not been any serious numerical simulations aimed at investigating the statistical properties of large scale flow in Rayleigh-Bénard systems. This is due to the fact that interesting statistical properties of large scale flow are observed (experimentally) only at relatively large Rayleigh numbers $R a, 10^{8}-10^{11}$, and after having collected data for rather long time periods (up to one year [ 6$]$ ). From a numerical point of view, major computer facilities are needed in order to properly resolve large $R a$ conditions 7] and simulating these flows for thousands of large eddy turnover times is, at present unfeasible. In this Letter, by using a suitable strategy, we show how to perform numerical simulations aimed at investigating the statistical properties of large scale flow in Raylaigh Bénard convection while maintaining the computational requirements at a reasonable level. The flow investigated in this Letter is that developing in a cylindrical cell of aspect ratio (diameter $d$ over cell height $h$ ) $\Gamma=d / h=1$ heated from below and cooled from above with an adiabatic side wall. All the surfaces boundary conditions are no-slip. 10 azimuthally equi-spaced numerical ideal probes are located on a circle halfway between the plates at a radius $r_{p}=0.2 h$; the "probes" provide simultaneous point-wise measurements of temperature and of the three velocity and vorticity components. The flow is solved by numerically integrating the three dimensional unsteady Navier-Stokes equations with the Boussinesq approximation:

$$
\begin{gathered}
\frac{D \mathbf{u}}{D t}=-\nabla p+\theta \hat{z}+\left(\frac{P r}{R a}\right)^{\frac{1}{2}} \nabla^{2} \mathbf{u}, \quad \nabla \cdot \mathbf{u}=0, \\
\frac{D \theta}{D t}=\frac{1}{(\operatorname{Pr} R a)^{\frac{1}{2}}} \nabla^{2} \theta,
\end{gathered}
$$

where $D / D t \equiv \partial_{t}+\mathbf{u} \bullet \nabla, \hat{z}$ the unity vector pointing in the opposite direction with respect to gravity, $\mathbf{u}$ the

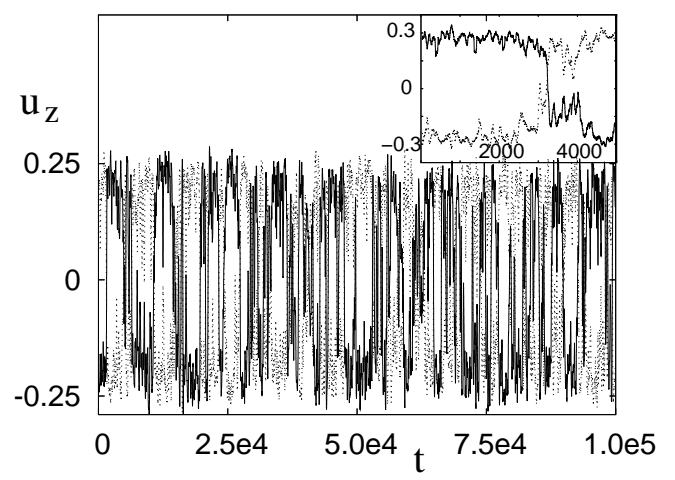

FIG. 1: Time evolution of the vertical velocity in one ideal probe. Many rather abrupt changes in the signal are observed. The numerical simulation are performed using (12) with an external random forcing on the temperature equation (2). In the inset we show the same quantity for the same value of $R a=6 \times 10^{5}$ without the random perturbation on the temperature field.

velocity vector, $p$ the pressure (separated from its hydrostatic contribution) and $\theta$ the non dimensional temperature. The equations have been made non-dimensional using the free-fall velocity $U=\sqrt{g \alpha \Delta h}$, the distance between hot and cold plates $h$ and their temperature difference $\Delta=T_{h}-T_{c}$; the non-dimensional temperature $\theta$ is defined $\theta=\left(T-T_{c}\right) / \Delta$ so that $0 \leq \theta \leq 1$. The above equations have been written in a cylindrical coordinate frame and discretized on a staggered mesh by central second-order accurate finite-difference approximations; the resulting discretized system is solved by a fractionalstep procedure with the elliptic equation inverted using trigonometric expansions in the azimuthal direction and the FISHPACK package [9] for the other two directions. The numerical method is the same as that described in [10] and [1] where further details of the numerical procedure can be found. The numerical experiments were performed at $P r=0.7$ and $R a=6 \times 10^{5}$ on a grid of $33 \times 49 \times 97$ nodes, respectively, in the azimuthal, radial and vertical directions; this grid was found to be sufficient in [11] for the used flow parameters. Owing to the relatively low value of $R a$, large scale flow reversals or reorentations are expected to occur on extremely long 


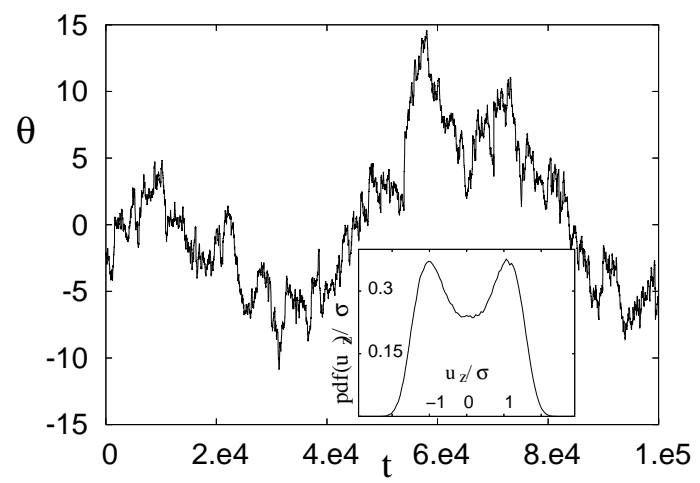

FIG. 2: Time evolution of the angular orientation $\Theta$ of the interface between warm rising fluid and cold falling fluid. In the inset we show the probability distribution of the vertical velocity as computed in one single probe.

time scales, too long to achieve an acceptable statistical convergence within a reasonable computational effort. In order to increase the turbulent fluctuations, one can either increase the value of $R a$ or alternatively decrease the Prandtl number (which is equivalent to increase the Reynolds Re number of the system), both methods implying an increase of numerical resolution. Alternatively, we can artificially increase thermal fluctuations by adding to the right hand side of (2) an extra forcing term $f$, $\delta$ - correlated in space and time. More precisely the explicit expression is $f=\epsilon \sqrt{\Delta t} \phi$ where $\epsilon$ is the amplitude of the perturbation, $\Delta t$ is the time step size and $\phi$ is a space dependent white-noise random number. The physical motivations for introducing $f$ are twofold: first of all, it has been pointed out ([8]) that small scale turbulent fluctuations can be quantitatively considered as an external random forcing acting on the large scale flow. Thus, one may consider $f$ as the overall effect of small "unresolved" turbulent motion for a smaller value of $\mathrm{Pr}$. Secondly, one can think that the effect of the external noise is to increase thermal diffusivity and, as a consequence, to increase $R e$ at fixed Nusselt number $N u$ Both arguments, however, have the same physical meaning. In this particular example the Nusselt number resulted $N u=4.1 \pm 0.1$ with and without the external noise on the temperature field, as expected, because the Nusselt is essentially independent of $\mathrm{Pr}$, i.e. on thermal diffusivity. In figure (11) we show the vertical velocity at one single ideal location as function of time for $\epsilon=0.1\left(T_{h}-T_{c}\right)$, while in the inset we show the same result for $\epsilon=0$. As we can see, a rather clear increase of the number of reversals is observed by using the external random forcing $f$. The value of $\epsilon \sim 0.1\left(T_{h}-T_{c}\right)$ has been chosen by trial and error, i.e. not too strong with respect to the deterministic dynamics and not too small to be irrelevant for the time scale of the reversal. As a consistency check, however, we have run similar simulations using half and twice the above value of $\epsilon$ obtaining similar statistics for the large scale flow. The main simulation was run for
$10^{5}$ time units that on account of the cell geometry correspond to about $10^{5} / \pi \approx 3 \times 10^{4}$ large eddy turnover times. Graphical inspection shows that the flow in the cell is roughly divided into two halves, one with a rising warm current (positive vertical velocity) and the other with cold sinking fluid (negative vertical velocity). The two regions are separated by an "interface" where the vertical velocity is close to zero. Because of the cylindrical geometry, the interface has no preferred orientation and, therefore, it is fluctuates in time. By using the same procedure described in [4] and [3] we can compute the instantaneous orientation $\Theta$ of the interface. As a consistency check we have applied the same procedure using either the temperature and the vertical velocity signals of the probes, always obtaining the same results. In figure (2), we show the time behaviour of $\Theta$, while in the inset we show the probability distribution of the vertical velocity as obtained by measuring it on a single probe. The behaviour of $\Theta$ is in close agreement with the experimental data of [4] and [3], while the probability distribution of the vertical velocity shows a bimodal shape in agreement with [1]. Next we can study the statistical properties of our large scale flow. In particular, the existence of a bimodal distribution for the vertical velocity on a single probe, allows us to study the statistical properties of the (random) switching time $(\tau)$ between the two states identified as the maxima of the probability shown in (2). More precisely, we define $\tau$ as the time interval between two successive zero-crossings of the vertical velocity at one ideal probe and we compute its probabity distribution $P(\tau)$. This study was first performed by [1] where an estimate of the vertical velocity was obtained by using the correlation time of two close temperature probes. In [1] and [3] $P(\tau)$ was found to be a power low for small enough $\tau$, while at large $\tau$ an exponential cutoff in $P(\tau)$ was observed. This is a rather peculiar result which is difficult to explain by using simple arguments based on the theory of stochastic differential equations or chaotic dynamics [8]. Our numerical simulations agree with the experimental results as shown in figure (3] circles). Following [3] we also computed the probability distribution $P_{\delta}$ of $\tau_{\delta}$ defined as the time between two events where both $\delta \Theta \equiv \Theta(t+\delta t)-\Theta(t)$ and $d \Theta / d t \equiv \delta \Theta / \delta t$ are larger than given thresholds; we have used for the thresholds the same values as [3] and have verified that the statistical results are robust with respect to the arbitrarily selected values. In the inset of figure 3 we show the log-lin plot of $P_{\delta}$ and compare it with an exponential distribution (line). As observed in the experimental analysis of [3], the probability $P_{\delta}$ seems to be fitted rather accurately by an exponential distribution. Figures (2) and (3) show our main results, i.e. our numerical simulations reproduce qualitatively and quantitatively the experimental findings. Next, we want to discuss briefly some theoretical ideas which may help in explaining the power law behaviour of the probability distribution $P(\tau)$ as computed by [1] and reproduced in our simulations. From a theoretical point of view, we 


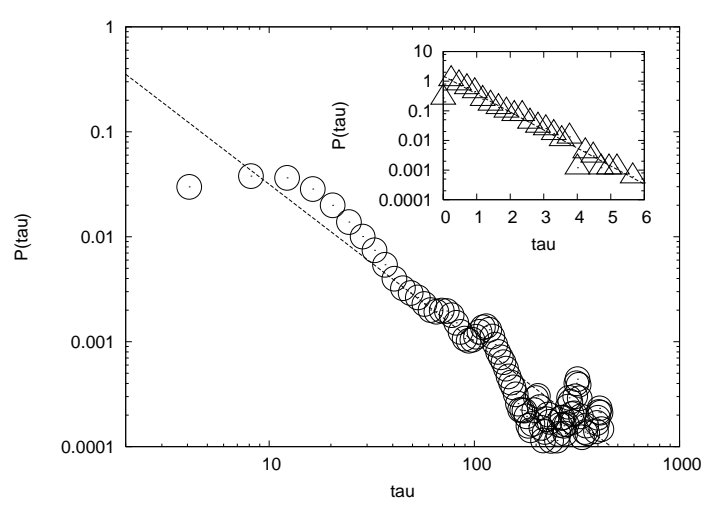

FIG. 3: Circles:log log plot of the probability distribution $P(\tau)$ of the random switching time $\tau$. Note that $P$ shows a power law behaviour shown by a straight line in the figure. Inset: $\log$-lin plot of the probability distribution $P_{\delta}\left(\tau_{\delta}\right)$ of $\tau_{\delta}$ following [3]. Note that the best fit is consistent with an exponential shape.

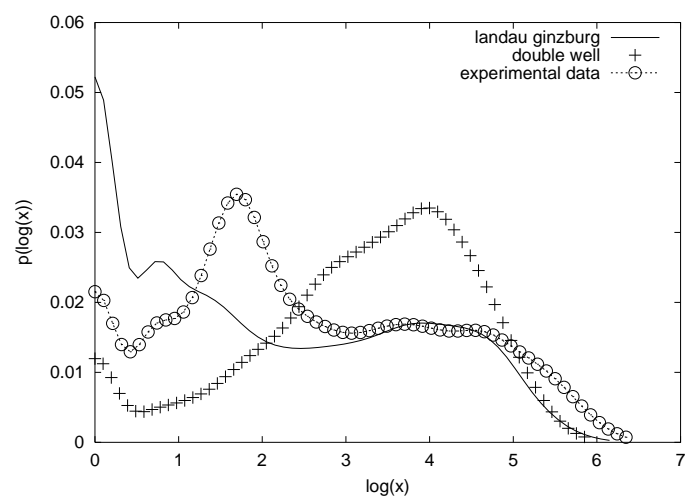

FIG. 4: Probability distribution of $\log (\tau)$ for the $\psi_{0}$ defined in (4) (line) as compared with the experimental data (circles) and the case of an overdamped brownian particle in a double well potential (see text for a description).

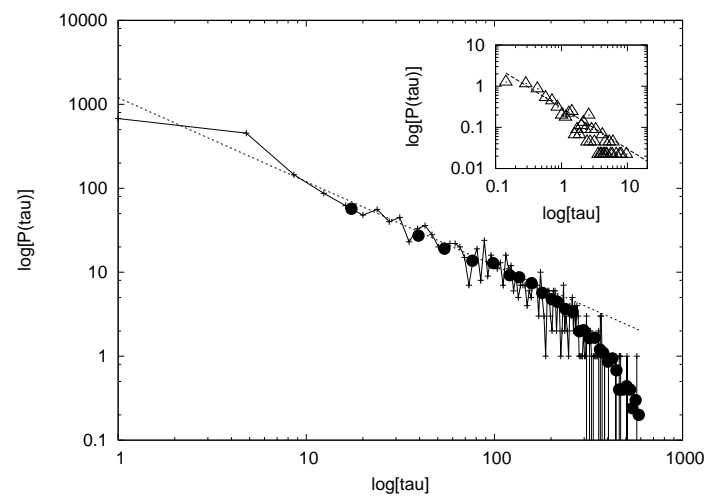

FIG. 5: Log-log plot of the probability distribution $P(\tau)$ of the random switching times $\tau$ between the maxima of $w$. Small black circles are obtained using (6) with $\Theta$ a random noise correlated in time. Line with symbol refers to the experimental data by [1]. In the insert, we show the same quantity computed with $\Theta$ obtained by our numerical simulations, see figure (2). can state the problem as follows: Let $Q$ be a random variable whose probability distribution is bimodal with maxima in $\pm Q_{0}$ and let $\tau_{Q}$ be the (random) switching time between the maxima. Is there any "simple" model for which $P\left(\tau_{Q}\right)$ shows a power law behaviour for small $\tau_{Q}$ ? We will show that such a model does exist and it may be relevant for thermal convection. To this aim, let us consider the Landau-Ginzburg equation (LGE) in two space dimensions for the field $\psi(x, y, t)$ :

$$
\partial_{t} \psi=m \psi-g \psi^{3}+\nu \Delta \psi+\sigma \eta(x, y, t)
$$

where $m, g, \nu, \sigma$ are real positive variables and $\eta$ is a white noise delta correlated in space and time. We assume $\psi$ defined on a periodic box of finite size $L$ and we indicate with $\langle. .\rangle_{s} \equiv L^{-2} \int . . d x d y$ the space average. We can decompose $\psi$ in its space average $\psi_{0} \equiv\langle\psi\rangle_{s}$ and a fluctuation $\phi$, i.e. $\psi \equiv \psi_{0}+\phi$. Then the equation for $\psi_{0}$ reads:

$$
\partial_{t} \psi_{0}=\left(m-3 g\left\langle\phi^{2}\right\rangle_{s}\right) \psi_{0}-g \psi_{0}^{3}+\sigma_{s} \eta(t)
$$

where $\eta$ is a white noise in time. More precisely, let us consider (3) defined on a regular lattice of $N \times N$ points of spacing $\Delta x=\Delta y=L / N$. Then $\sigma_{s} \equiv \sigma / N^{2}$ and we assume $\sigma_{s}=$ const. for any $N$ (see [13] for a correct definition of the problem). It is worth reminding that the equilibrium probability distribution of (3) has been widely used as a model of second order phase transition in the infinite volume limit. For a suitable choice of the parameters $m, g$ and $\nu$, the term $\left\langle\phi^{2}\right\rangle_{s}$ can be large when $\psi_{0}$ becomes small, i.e. the system at $\psi_{0} \sim 0$ shows strong fluctuations as it is usual in second order phase transitions. In this case, the linear term in (4), i.e. $\left(m-3 g\left\langle\phi^{2}\right\rangle_{s}\right)$, can occasionally change sign and the switching between the two maxima of the probability distribution of $\psi_{0}$ can be more frequent. This is equivalent to say that the switching time is no longer controlled by the mechanism leading to an exponential distribution and one can observe a scale (in time) invariant probability distribution, i.e. a power law. In figure (4) we show the probability distribution of the switching time of $\psi_{0}$ obtained by numerically integrating equation (3), for $N=32, \nu=0.1, m=1, g=9$ and $\sigma_{s}=0.05$. In particular we plot the probability distribution of $\log (\tau)$, where $\tau$ is the random switching time computed by using the parameter $\psi_{0}$. Note that if $P(\tau) \sim \tau^{-1}$ then $P(\log (\tau)) \sim$ const. In the same figure we show the probability distribution of $\log (\tau)$ for the experimental data by [1]) and for the well known case of an over-damped Brownian particle in a double well potential with the same parameter $m$ and $g$ and stochastically perturbed by a white noise with variance $\sigma_{s}$. In the last case, we know that the probability distribution of $\tau$ is exponential and, indeed, in figure (4), $P(\log (\tau))$ for the double well potential is not constant. On the other hand, $P(\log (\tau))$ is constant for almost one decade for the numerical simulation of (3) using $\psi_{0}$ as order parameter. Thus, we may tentatively state that the equation (3), and in particular (4), can be 
considered as a conceptual model for the experimental results of [1]). From a physical point of view, we remark that there exists a non trivial feedback between the variance of the fluctuations in space and the value of the order parameter $\psi_{0}$. It is now tempting to understand whether the same "physical" mechanism can be observed in our numerical simulation of the Rayleigh-Bénard turbulence. According to our previous discussion, the overall picture which emerges is quite simple and agrees with the original description of [4]. As a first approximation we can think that the large scale flow rotates randomly in time with some orientation $\Theta(t)$ performing a random walk in the interval $[0,2 \pi]$. The vertical velocity in a single point is almost insensitive to $\Theta$ unless the interface is close to the ideal probe. A suitable model for $\Theta(t)$ can be build as follows. Let us consider two variables $x_{1}=r \cos (\theta)$ and $x_{2}=r \sin (\theta)$ which satisfy the following equation

$$
\frac{d x_{i}}{d t}=-\frac{\partial V}{\partial x_{i}}+\text { noise }
$$

where $V \equiv-1 / 2 r^{2}\left(R^{2}-r^{2}\right)$ and $r^{2}=x_{1}^{2}+x_{2}^{2}$, i.e. $r \sim R$ for most of the time. We can imagine that $\theta$ is precisely the variable $\Theta$ describing the interface direction of the large scale wind. Then denoting by $w$ the value of the vertical velocity, a qualitative model of $w$ as a function of $\theta(t)$ is simply given by:

$$
w=\frac{x_{2}}{\sqrt{x_{2}^{2}+A}}=\frac{\sin (\theta)}{\sqrt{\sin ^{2}(\theta)+A / r^{2}}}
$$

where the parameter $A$ is a measure of the interface thickness, whose fluctuations are controlled by $r$. Using (6) and our simple model, one can easily show that:

$$
\frac{d w}{d t}=a w-(a+b A) w^{3}+\text { noise }
$$

where $a \equiv R^{2}-2 x_{1}^{2}$ and $b=2 . . \quad$ Equation (7) has a form of an over-damped Brownian particle in a double well potential. However the term $a$ can change sign (exactly as in (4)) because of the (strong) fluctuations of $x_{1}$. In order to compute the probability distribution of the switching time $\tau$ between the two maxima in the probability of $w$, we have numerically integrated equation (5), with $R=1, A=0.1$ and the variance of the noise equal to 0.1. The final result is shown in figure (5)) (small black circles) and compared with the experimental finding of 1] (line with symbol). As we can see $P(\tau)$ is close to a power law with slope -1 in agreement with the experimental findings of [1]. In the same figure, we show the probability distribution $P(\tau)$ computed by using the approximate value $w \sim \sin (\Theta) / \sqrt{\sin ^{2}(\Theta)+A}$ and the value of $\Theta$ obtained in the numerical simulation (see figure (2)). A good agreement is observed with a power law $P(\tau) \sim \tau^{-1}$. Thus (6) seems to capture the physical reason for the observed numerical and experimental results in the statistical properties of flow reversal. In summary, we have shown that the statistical properties of large scale flow in Rayleigh Bénard convection can be efficiently simulated by using an external random forcing in the temperature field. The effect of the forcing is to increase thermal diffusivity, i.e. to decrease the Prandtl number. Consequently, the effective Reynolds number achieved in this way is larger with respect to the one without external forcing. Our numerical simulations agrees qualitatively and quantitatively with the experimental findings of [1] , [3] and [4]. Finally, we have discussed some theoretical ideas which may be able to explain the statistical properties of flow reversals.

We acknowledge K.R. Sreenivasan, G. Alhers, K.Q. Xia, T. Sato, L. Kadanoff, J. Niemela, for useful discussions.
[1] K.R. Sreenivasan, A. Bershadskii and J.J. Niemala, Phys. Rev. E, 65, 056306 (2002); K.R. Sreenivasan, A. Bershadskii and J.J. Niemala, Physica A, 340, 574579, (2004);R.C. Hwa, C.B. Yang, A. Bershadskii, J.J. Niemela and K.R. Sreenivasan, Phys. Rev. E, 72, 066308$1-5$ (2005).

[2] Xi, Zhou and Xia, Phys. Rev. E 73, 056312, (2006), C. Sun, K.-Q. Xia, and P. Tong, Phys. Rev. E. 72, 026302 (2005)

[3] E. Brown, A. Nikolaenko and G. Ahlers, Phys. Rev. Lett., 95, 084503 (2005)

[4] S. Cioni, S. Ciliberto and J. Sommeria, J. Fluid. Mech., 335, 111 (1997)

[5] F. Fontenele Araujo, S. Grossmann and D. Lohse, Phys. Rev. Lett., 95, 084502 (2005)
[6] E. Brown and G. Ahlers, J. Fluid Mech., (2006) To appear

[7] G. Amati, K. Koal, F. Massaioli, K.R. Sreenivasan and R. Verzicco, Phys. of Fluids, 17, 121701 (2005)

[8] R. Benzi, Phys. Rev. Lett., 95, 024502 (2005).

[9] P.N. Swartzrauber, SIAM J. Numer. Anal., 11, 1136, (1974).

[10] R. Verzicco and P. Orlandi, J. Comput. Phys. 123, 402, (1996).

[11] R. Verzicco and R. Camussi, J. Fluid Mech. 477, 19, (2003).

[12] R. Benzi, A. Sutera, J. Phys. A: Math. Gen. 37,L391L398, (2004).

[13] R. Benzi, G. Jona-Lasinio, J. Stat. Phys.,55,508, (1988) 